\title{
Cooling/Congealing Particle Sizing
}

National Cancer Institute

\section{Source}

National Cancer Institute. Cooling/Congealing Particle Sizing. NCI Thesaurus. Code C113038.

Formation of particles by solidification of dispersed droplets within a cooler gas. 